\begin{tabular}{|c|c|c|}
\hline-9 & $\begin{array}{c}\text { European Association for the } \\
\text { Development of Renewable Energies, Environment } \\
\text { and Power Quality (EA4EPQ) }\end{array}$ & $\begin{array}{l}\text { International Conference on Renewable Energies and Power Quality } \\
\text { (ICREPQ'12) } \\
\text { Santiago de Compostela (Spain), 28th to 30th March, } 2012\end{array}$ \\
\hline
\end{tabular}

\title{
Performance Assessment of an Aeolian Roof for the Exploitation of Wind Power in Urban Areas
}

\author{
S. Carcangiu, A. Montisci \\ Department of Electrical and Electronic Engineering \\ University of Cagliari \\ Piazza d'Armi - 09123 Cagliari (Italy) \\ Phone number:+0039 0706755871 e-mail: s.carcangiu@diee.unica.it, amontisci@diee.unica.it
}

\begin{abstract}
In this work, an innovative system for depletion of wind power in urban areas is presented. The energy conversion system is installed in the place of the roof of the civil building, carrying out the same covering functions, but at the same time it conveys the air flow towards a vertical axis centripetal turbine. The studied system makes it possible to avoid some typical drawbacks of the eolic generation, such as the encumbrance of the blades, noise, cut-in and cut-off velocities of the wind, presence of turbulence. Due to these problems, the wind energy usually is not considered as a suitable energy source for the urban areas. In this work, a study has been performed in order to assess the potentialities of the proposed system for fulfilment of energy demands in urban areas. By means of a finite element analysis, the sensitivity with respect to the design parameters will be evaluated.
\end{abstract}

\section{Key words}

Wind power in urban areas, Stator, Aeolian Roof, Computational Fluid Dynamics (CFD).

\section{Introduction}

As it is well known, wind and sun are the most promising renewable energy sources, both for the concentration and availability [1]. Typically, the wind energy is preferred in extra urban areas, because of the better ratio between produced energy and used land with respect to other renewable energy sources, whereas the depletion of this energy source is almost zero in urbanized areas. This is due to several reasons, such as urban constraints, high turbulence triggered by neighbouring buildings and the difficulty to re-orienting the generator on the basis of the wind direction $[2,3]$. In spite of these problems, one could be interested to exploit wind energy in urban areas, both to integrate the energy produced by the sun, and to increase the continuity of production, so that the energy to store or to exchange with the electric network can be reduced.

The system presented in this paper aims to solve the cited drawbacks of wind generation in order to make possible its extensive use in urban areas. The generation system substitutes the roof of the building carrying out the same covering function. It consists of an external static structure, the stator, whose task is conveying the flow towards the centre of the roof, where a vertical axis centripetal turbine transforms the wind energy in mechanical energy. Such a layout of the system entails several advantages. Firstly, the visual impact and safety problems of the turbine are definitively avoided, because it is hidden inside the structure. Secondly, the wind direction is not a problem, because the vertical axis of the rotor allows it to capture the wind no matter which is the provenance direction. Thirdly, the stator allows one to intercept a wide section of flow, even if the dimension of the turbine is very small, depending on the ratio between the inlet and the outlet cross section of the stator. This allows one to exploit a wider range of wind velocities with respect to common wind generators, because of a lower cut-in and unlimited cut-off velocities.

A laboratory-scale prototype of the system has been realized in order to perform a sensitivity analysis with respect to the design parameters of the system. In this work, a study is performed in order to evaluate the performance of the system for different scenarios. A Computational Fluid Dynamics (CFD) analysis has been performed and different solutions have been tested in order to optimize the set of design parameters. The paper is organized as follows: The present paper is organized as follows. In Section 2 the layout of the system is described. In Section 3 the 2D numerical model of the system and the equations describing the physical phenomenon are reported. In Section 4 two parametric studies are performed in order to determine the best number of stator ducts and the best ratio between inlet and outlet section of the stator. In Section 5 the results of an optimized structure are discussed. Some conclusions will end the paper.

\section{System Layout}

Fig. 1 shows the 3D model of the studied system. The prototype is composed by a fixed part, the stator, with cylindrical shape and outer diameter equal to $1 \mathrm{~m}$. 


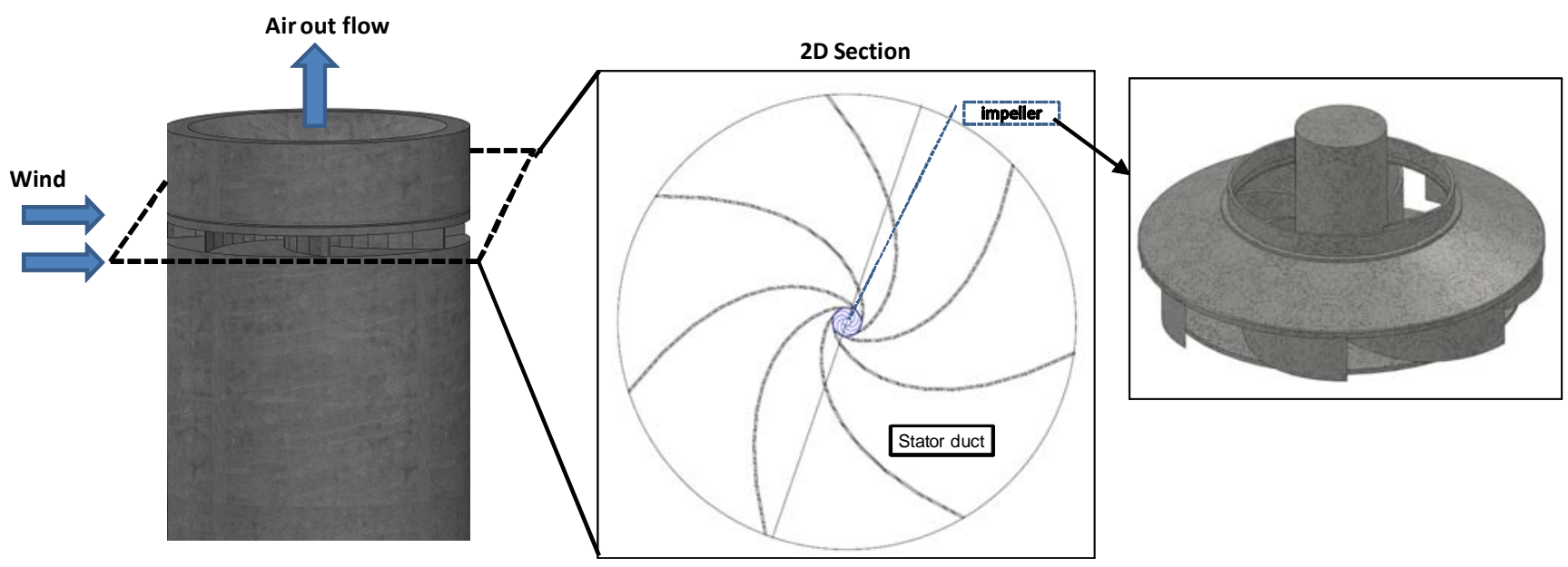

Fig. 1. 2D and 3D model of the system studied: stator ducts and impeller.

The moving part, the impeller, is a vertical axis centripetal turbine, with 7 blades. The turbine is a commercial product, so that in designing the diameter of the turbine the choice is limited to the catalogue values. In this case, the diameter of the impeller is equal to $12 \mathrm{~cm}$ and the inlet section of its ducts is equal to $5 \times 1 \mathrm{~cm}^{2}$. The stator blades have a helicoidal shape, so that a great ratio between the two terminal sections of the duct can be achieved.

The rate of convergence of stator duct is a crucial design aspect. In fact, a great ratio between the two terminal cross sections implies that a wide tube of flux is captured, but it is processed by a small turbine, in this way obtaining advantages both in terms of cut-in and cut-off velocities of the wind. In fact, the friction and the inertia are smaller, then the small turbine can get to rotate even for very small wind velocities.

In turn, in the presence of strong wind, the centrifugal strength is strongly dependent on the diameter of the impeller, so that a small turbine can carry high rotating velocities. On the other hand, part of the wind hinting the building bypasses it, and the ratio between the bypassing flow and the one evolving in the system depends on the reciprocal fluid dynamic impedance. For this reason, the designer has to find a crossover point between the two conflicting exigencies of having a small turbine and capturing a great quantity of energy.

The best compromise should be evaluated on the basis of economic, legislative and environmental aspects, which are beyond the scope of this paper, therefore we will limit to evaluate the sensitivity of performance with respect to the main design parameters.

The design parameters we use are the diameter of the turbine and the number of stator blades, both affecting the ratio between the terminal sections of the stator ducts, whereas the external dimensions of the building are assigned. In the section 4 several solutions have been studied for the shape and the number of ducts of the stator. The outlet flow of the turbine has vertical direction and it escapes through a divergent duct, at the end of which the exhaust flow intersects the bypassing flow. The divergent duct allows one to increase the velocity of the air in the turbine, and the depression on the top of the building contributes to reduce the impedance of the internal path of the air.
The impedance of the bypassing flow can be increased by means of mouldings in the facade of the building, but these further possibilities to improve the performance of the system have not been explored in this work.

\section{System Modelling}

The bi-dimensional cross section shown in Fig. 1 has been modelled and the commercial software Comsol Multiphysics ${ }^{\circledR}$ 4.2a [4], which implements the CFD analysis, has been used to solve the model equations.

The wind flow around and inside the building model is described by the Navier-Stokes equations [5], that have been solved for the velocity field and the pressure. Considering an incompressible flow and a stationary study, the nonlinear system of equations is the following:

$$
\begin{aligned}
& \rho \mathbf{u} \cdot \nabla \mathbf{u}-\nabla \cdot\left(\mu\left(\nabla \mathbf{u}+(\nabla \mathbf{u})^{T}\right)\right)+\nabla \cdot \mathbf{p}=\mathbf{F} \\
& \nabla \cdot \mathbf{u}=\mathbf{0}
\end{aligned}
$$

where $\mu$ denotes the dynamic viscosity, $\mathbf{u}$ the velocity vector, $\rho$ the density of the fluid, $p$ the pressure and $\mathbf{F}$ is a body force term.

The modeled fluid is air with viscosity $10^{-5}\left[\mathrm{~N} \cdot \mathrm{s} / \mathrm{m}^{2}\right]$ and density $1.2 \mathrm{~kg} / \mathrm{m}^{3}$. The first equation is the momentum balance, and the second is the equation of continuity for incompressible fluids.

The following boundary conditions are applied in the computational domain (Fig. 2):

- the inlet and the outlet conditions are set on the opposite boundaries;

- an additional outlet condition is set on the inner boundary of the impeller in order to simulate the outlet flow of the turbine;

- $\quad$ assuming the distribution of wind velocities during the year provided by statistical public institutions [6], the inlet velocity vector is set normal to the boundary and equal to $\mathbf{u} \cdot \mathbf{n}=5[\mathrm{~m} / \mathrm{s}]$;

- the Outlet boundary condition sets the pressure to atmospheric value of $p_{0}=1[$ Bar $]$; 


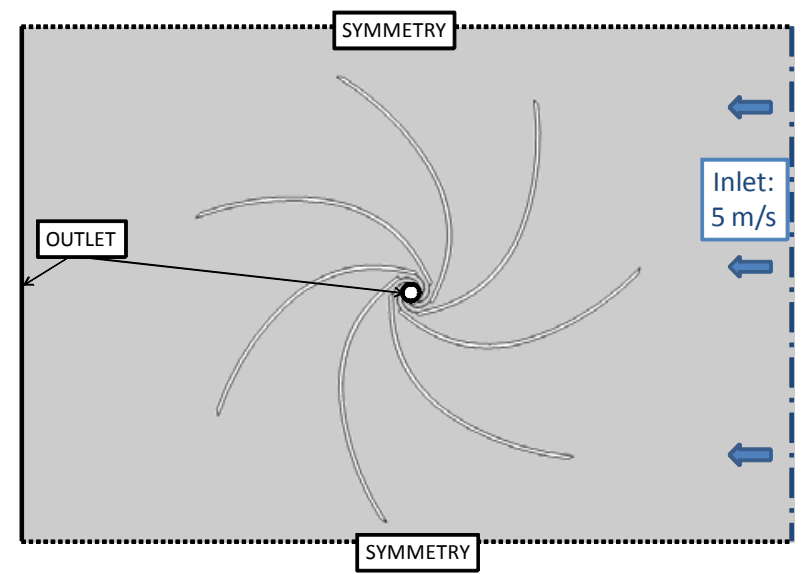

Fig. 2. Bidimensional cross section modelled.

- the No-slip boundary condition, that eliminates all components of the velocity vector $(\mathbf{u}=0)$, is set on the walls of the stator ducts and of the impeller;

- a Symmetry condition, applied to all the remaining boundaries, states that the component of velocity perpendicular to the boundary is zero.

Several analyses have been performed, with different values of design parameters, to which correspond a different ratio between the inlet and the outlet sections of the stator duct and a different number of ducts. The parametric analysis has been done considering that the wind pressure generates a torque that forces the impeller to rotate.

The value of the torque $\boldsymbol{T}$ has been calculated by integrating over the "boundary" of the impeller the momentum due to the pressure $\boldsymbol{P}$, equal to:

$$
T=\int_{A}\left[\left(P_{x} \cdot y\right)-\left(P_{y} \cdot x\right)\right] d A \quad[N \cdot m]
$$

where $\mathbf{A}$ is the surface of the impeller blades.

The available power $P$ of an air flow crossing the section $A$ (measured in $\mathrm{m}^{2}$ ) is equal to:

$$
P=\frac{1}{2} \int_{A} \rho \cdot U^{3} d A
$$

where $P$ is the power, $\rho$ is the density of the air and $U$ is the velocity of the wind.

As the power is proportional to the cube of velocity, the reduction of flow due to a decrease of the minimal section can be partially compensated by the velocity increase.

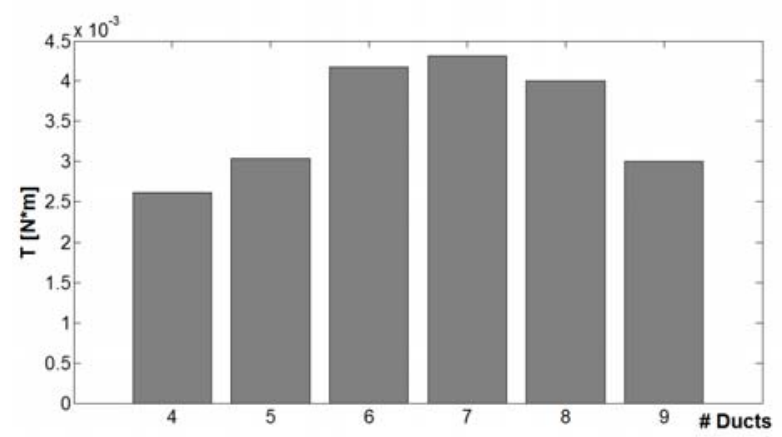

Fig. 3. Mean value of the torque for different number of the stator ducts.

\section{Parametric analysis}

In the following, different simulations has been analysed considering the number of ducts of the stator and the ratio between the inlet and the outlet sections of the stator duct, hence a different dimension of the turbine, as design parameters. Several scenarios that correspond to different positions of the impeller in order to calculate a mean value of the torque and of the power have been evaluated.

\section{A. Number of blades}

We can expect that the number of stator blades to which corresponds the best matching, is the same number of the impeller blades. Nevertheless, by reducing the number of stator blades we reduce the loss of energy due to friction between the air and the walls. At the same time, we are interested to reduce the complexity on the structure. An analysis has been performed by varying the number of stator blades from 4 to 9 with a fixed ratio between the diameters of the stator and the rotor, and the corresponding torque exercited of the impeller has been calculated.

Fig. 3 describes the obtained results. As one can see, there is a clear tendency of the torque to be maximum when the number of blades of the stator is the same of that of the rotor. The Fig. 4 shows a further interesting result. The produced power has been evaluated by integrating the formula (3) on the outlet surface of the stator and multiplying the result for a typical efficiency value of centripetal turbines [7] $(\eta=0.8)$ and the height of the duct $(h=1 \mathrm{~cm})$. The advantage to assume the same number of blades in the stator and in the impeller is here more evident. We find that encreasing the number of stator blades in general gives rise to a reduction of the power, as one can expect because of a more extended boundary layer, but having the same number of blades in the stator and in the rotor clearly favours the flow of the air and as a consequence gives rise to a higher efficiency. On the basis of the above described results, a number of blades equal to that of the impeller has been chosen for the stator too.

\section{B. Diameter of the impeller}

A series of analyses has been performed on the system with 7 stator blades, obtained in the previous analysis, by

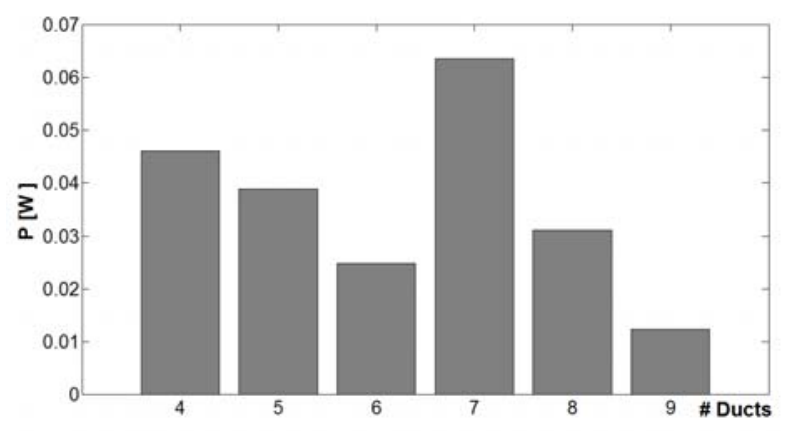

Fig. 4. Mechanical power generated by the turbine for different number of the stator blades. 


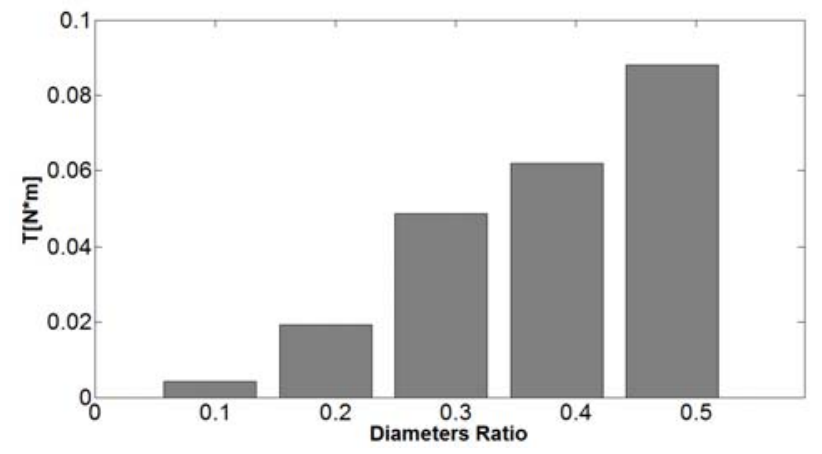

Fig. 5. Mean value of the torque for different ratio between the inlet and the outlet sections of the stator duct.

varying the diameter of the impeller, for a fixed external diameter of the stator. Several phenomena affect the variation of efficiency when we modify the ratio between the stator and the impeller diameters. In fact, on one hand if the impeller diameter is huge, the length of the stator duct is short and then the losses are reduced, but at the same time, the wider the impeller the greater is the friction due to bearings. Furthermore, the aim of this study is reducing the dimension of the impeller as much as possible, for both installation and maintenance costs of the system.

Figures 5 and 6 show the results obtained by the parametric analysis of the turbine. As expected, both torque and generated power rise with the dimension of the impeller, but while the torque rising is quit linear, the generated power follows a more irregular trend. As it can be seen, there is an advantage to duplicate the diameter of the impeller with respect to the reference size, used in the previous parametric analysis, whereas we find a reduction of the power when we adopt an impeller diameter which is 3 or 4 times the reference value. Suddenly, the power gets to increase passing from 4 to 5 times the reference value. Anyway, this last solution cannot be considered as suitable. In fact, the prototype under study aims to model the roof of a building, so that choosing a diameters ratio equal to 0.5 means that the rotor of the system has a diameter equal to one half of the minor side of the building. To take the final decision on the design parameters of the systems we will need to know the requirements of energy of the building and the probability density function of the wind.

\section{Results}

In this section, an analysis has been performed by assuming to have a building with a $10 \times 10 \mathrm{~m}^{2}$ plant and energy consumption per year equal to $2000 \mathrm{kWh}$ (four persons). A stator having 7 blades and a ratio between stator and rotor diameters equal to 5 has been assumed. In general the wind speed is described by the two parameters Weibull distribution [8], whose probability density function (pdf) is given by:

$$
f(U)=\frac{k}{\lambda}\left(\frac{U}{\lambda}\right)^{k-1} \exp \left[-\left(\frac{U}{\lambda}\right)^{k}\right]
$$

where $k$ is the shape parameter, $\lambda$ the scale parameter and $U$ the wind speed. In fig. 7 the Weibull pdf histogram for

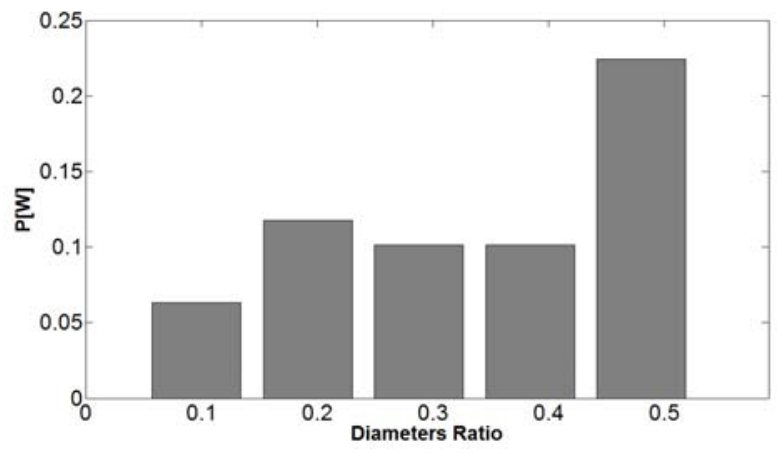

Fig. 6. Mechanical power generated by the turbine for the stator-rotor diameters ratio.

the average $10 \mathrm{~m}$ wind speed distribution in Sardinia, a region of Italy, is shown. This plot has been obtained considering respectively $k=1.44$ and $\lambda=6.5[\mathrm{~m} / \mathrm{s}]$ as Weibull parameters [9].

The total energy $\mathbf{E}$ produced by this aeolian roof in a time period $\mathbf{T}$ (one year, in this paper), is calculated considering the instantaneous power $\mathbf{P}$ obtained by the numerical simulations by the discrete relation:

$$
E=\sum_{i=1}^{25} P_{i} \cdot \Delta t
$$

The obtained total energy is a cumulative value given by the sum of the energy contributions in defined time intervals. Such time intervals are deduced on the basis of the wind speed frequency distribution diagram in Fig. 7. The obtained value of $\mathbf{E}$ is equal to $448.4 \mathrm{KWh}$.

As it can be seen, the energy production of the system is less of the required by the hypothetic demands. Actually, the aim of this work is only to assess the procedure we could use to optimize the design of the system, taking into account the requirements and the averaged weather conditions of the specific region where the system has to be implemented. The system offers many possibilities to increase the efficiency of the system and the quantity of produced energy, simply by modifying the layout of the stator. In fact a greater ratio between inlet and outlet cross sections of the stator can be obtained by narrowing the stator duct along both vertical and horizontal direction, in this way increasing the ratio between inlet and outlet cross section of the stator ducts. Another possibility is assuming a square plant of the building, in order to increase to impedance of the external path of the flow, but on the other hand, the prevalent direction of the wind becomes more important than in the case of circular

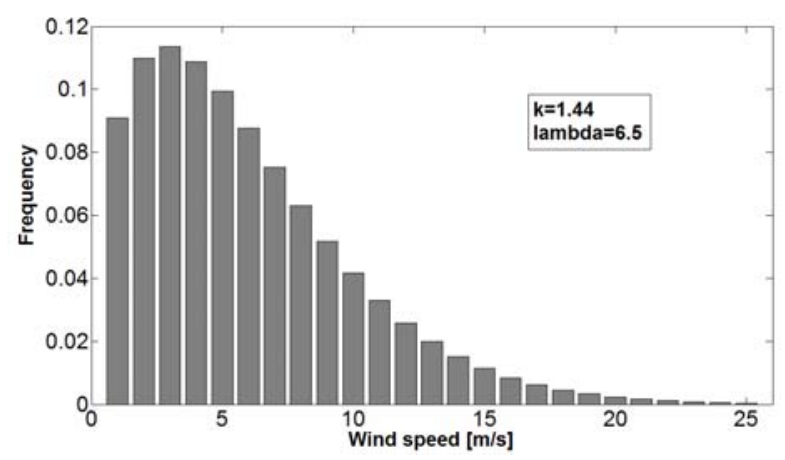

Fig. 7. Weibull pdf of the distribution of the wind speed during one year in Sardinia. 


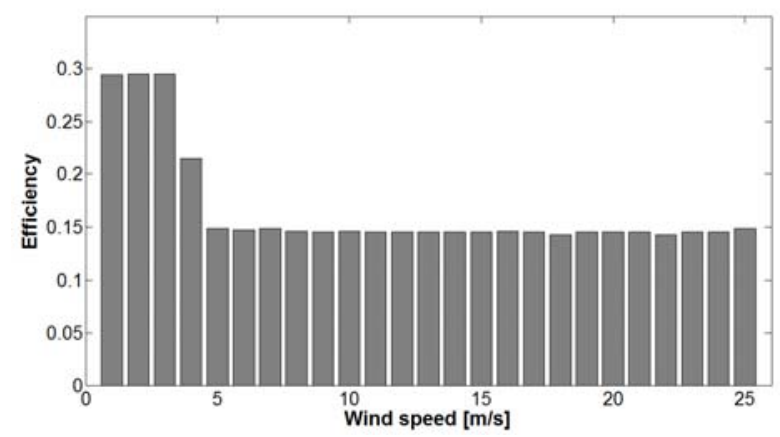

Fig. 8. Sistem efficiency for the wind speed.

plant, where instead the direction of the wind in practice does not affect the efficiency of the system.

As a measure of system efficiency, the ratio between the power evolving in the turbine and the available power will be evaluated. As available power, that one of a tube of flow without obstacles with a cross section equal to the inlet cross section of the system will be considered. This value is less than 1 and it can be interpreted as the cost of having a turbine whose inlet section is smaller than the inlet section of the stator. In Fig. 8 the efficiency of the studied system for different wind velocities is reported. As can be noted, such efficiency is higher for very low wind velocities, decreases at about $4 \mathrm{~m} / \mathrm{s}$ and assumes a quite constant trend for greater velocities. This behavior reflects the fact that a transition from laminar to turbulent flow takes place around said velocity of the wind. Obviously, the production of energy will by very higher at high velocities, because against a reduction of efficiency at one half, the power grows with the cube of the velocity.

Finally, Fig. 9 shows the velocities map for the adopted design solution, with the hypothesis of a wind velocity equal to $5 \mathrm{~m} / \mathrm{s}$. As it can be seen, the stator makes it possible to increase the wind velocity up to $10 \mathrm{~m} / \mathrm{s}$, in this way concentrating the power of the wind in a limited area, allowing one to use a turbine inlet section which is $1 / 5$ of the involved flow tube which hints the stator inlet section.

\section{Conclusion}

An urban-use-oriented wind generation roof has been presented in this paper. The proposed system makes it suitable depleting the wind energy in built-up areas. In fact, with respect to typical eolic generation systems, in the here proposed the turbine is in-shore, because it is contained inside a flow conveyor which performs at the same time as stator of the turbine and as roof of the building. This allows one to employ turbines which crosssection is small with respect to the elaborated flow tube, in this way saving installation costs and increasing the functioning range of admitted wind velocities. As is used to do in urban areas, a vertical axis turbine is adopted, so that the wind direction does not affect the efficiency of the system, nor any reorienting apparatus is necessary. Preliminary analyses on a prototype suggest that hardly the proposed system could satisfy the energy demands of a common building; anyway several design parameters

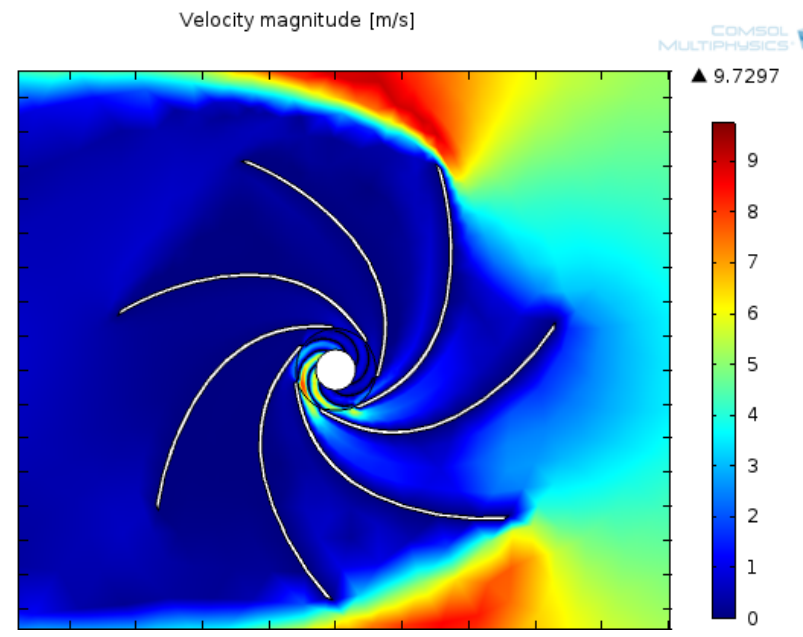

Fig. 9. Velocity map of the 2D model.

could be exploited in order to increase both its efficiency and productivity. In perspective, an engineered system could be suitably integrated in a new building and could give a meaningful contribution to satisfy the energy demands of buildings inserted in built-up areas.

\section{Acknowledgement}

This work is supported by the operating program of Regione Sardegna (European Social Fund 2007-2013), L.R.7/2007, "Promotion of scientific research and technological innovation in Sardinia"

\section{References}

[1] J. F. Manwell, J. G. McGowan, A. L. Rogers, Wind Energy, Theory, Design and Applications, Contract NAS2-11665, Muadyne Report 83-2-3, John Wiley and Sons, pp. 154-162, (2006)

[2] S. Eriksson, H. Bernhoff, M. Leijon, Evaluation of different turbine concepts for wind power, Renewable and Sustainable Energy Reviews, vol. 12, no. 5, pp. 1419-1434, (2008)

[3] A.S. Bahaj, L. Myers, P.A.B. James, Urban energy generation: influence of micro-wind turbine output on electricity consumption in buildings, Energy and Buildings, 39 2, pp. 154-165, (2007)

[4] http://www.comsol.com

[5] R. Temam, Navier-Stokes equations, theory and numerical analysis, AMS-Chelsea Series, AMS, Providence, (2001)

[6] L. Baggini, M. Benini, G. Botta, C. Casale, C. Cavicchioli, General Assessment of exploitable Wind Resources in Italy, Proc. of Int. Conf. on Clean Electrical Power 2007, pp. $605-612,(2007)$

[7] S.L. Dixon, B. Eng, Fluid Mechanics and Thermodynamics of Turbomachinery (5th Edition), Elsevier (1998)

[8] Seguro, J.V., Lambert, T.W., "Modern estimation of the parameters of the Weibull wind speed distribution for wind energy analysis", J Wind Eng. Ind. Aerodyn (2000), 85(1):75-84

[9] F. Cassola, M. Burlando, L. Villa, P. Latona and C.F. Ratto, Evaluation of the Offshore Wind Potential along the Italian Coasts, Owemes, Citavecchia, Italy, 20- 22 April, 2006 